\title{
A comparison of manufacturing and disassembly systems from a cellular configuration point of view
}

\author{
Julio Mar-Ortiz \\ Faculty of Engineering, Universidad Autónoma de Tamaulipas \\ 89140 Tampico Tamaulipas, Mexico \\ E-mail: jmar@uat.edu.mx* \\ *corresponding author \\ Belarmino Adenso-Díaz \\ Engineering School, Universidad de Oviedo \\ 33203 Gijón, Spain \\ E-mail: adenso@epsig.uniovi.es \\ José Luis González-Velarde \\ Tecnológico de Monterrey \\ 64849 Monterrey, Nuevo León, Mexico \\ E-mail: gonzalez.velarde@itesm.mx
}

\section{Paper published by the journal:}

International journal of Advanced Manufacturing Technology (2015), Vol. 79,

pp: 2003-2016 (doi: 10.1007/s00170-015-6969-6)

\begin{abstract}
The main aim of this research is to analyze the differences between cellular manufacturing and cellular disassembly systems. While doing so, this paper makes a relevant contribution. It proposes a mathematical programming formulation for robust manufacturing and disassembly cell formation problems. A solution method based on the Tabu Search schema is adapted for both problems, and will be used for the comparison analysis. The fact that the Tabu Search algorithm has been effective in different cell formation approaches gives us confidence in its reliability, and although alternative metaheuristic approaches may be developed in the future, we opted for adapting this algorithm to the problem at hand, since our first aim is to obtain data to analyze the difference in the design of both types of production environments and not to develop new approaches to the cell formation problem. A full factorial design study is proposed in order to appraise the significance of controllable factors in cellular designs. Four dependent variables were considered in the
\end{abstract}


experiments: total cost, size of the cells, number of intercellular moves, and machine utilization. The issue here is to see what happens with the solutions for each production environment (manufacturing and disassembly) when varying factors, in order to analyze the differences and similarities between both.

Keywords: cell formation problem; cellular manufacturing; disassembly; robust optimization; operational research.

\section{Introduction}

The cell formation problem (CFP) has been one of the most studied problems in manufacturing literature, and is the first technical problem faced in the design of a Cellular Manufacturing System. The CFP consists of grouping machines (and eventually tools, storages, operators, etc.) into manufacturing cells as independently as possible in order to satisfy a performance criterion, for example the inter-cell traffic or the number of inter-cell movements. To address this problem, several and diverse solution procedures have been developed and published over recent years. Wemmerlöv and Hyer [1], Singh [2], Selim et al. [3] and Papaioannou and Wilson [4] provide extensive reviews of prior research. Recently, the growing interest in Environmentally Conscious Manufacturing and reverse logistics has motivated a renewed attention to disassembly systems. Of particular interest to our research is the idea of extending the benefits of a cellular configuration into the disassembly process [5-6].

There is a vast literature on disassembly systems [7-10], which can be widely classified into three major areas [10]: 1) modeling and representation of product disassembly sequences, 2) disassembly process planning, which includes the extent to which disassembly should be performed and how to decide the optimal disassembly sequence [11], and 3) disassembly system design and line balancing.

The design of a cellular configuration for the disassembly process or the disassembly cell formation problem (DCFP) belongs to the third category. To the best of our knowledge, the first reference regarding the DCFP from an analytical point of view is found in Adenso-Díaz et al. [5], who present a mixed integer programming formulation that minimizes the total cost function of 
machinery depreciation and intercellular movement of products. The proposed model seeks to group the disassembly tasks and assign them to cells together with their required resources so that total costs are minimized. Based on such formulation, Mar-Ortiz et al. [6] define a set of neighborhood structures for the problem, which are implemented in a Tabu Search schema to analyze their behavior in a real-world instance. Andrés et al. [12] propose a two-phase approach for determining the optimal disassembly sequence when the disassembly system has a cellular configuration. Finally, Mar-Ortiz et al. [13] propose a variable neighborhood search (VNS) algorithm to solve a disassembly cell formation problem with demand variability under a reconfigurable approach. The reconfigurable approach allows the cells to be rearranged periodically to deal with demand variability in a multi-period planning horizon.

The main assumption of these papers is that the benefits of any type of layout are the same for similar production environments, independently of the operations to be performed. When only one type of product is to be processed and the volume is high, a dedicated disassembly line seems to be justified. However, disassembly systems are characterized by the arrival of several similar types of product to be dismantled, each one with a low-medium volume. Therefore, if we consider the use of a cellular configuration to take into account the similarities in the disassembly tasks, the disassembly operations may be improved, as happens with manufacturing processes that have the same characteristics.

As regards the problem of selecting a suitable manufacturing layout for a given production system, Gamberi et al. [14] proposed an analytical model to evaluate the suitability of implementing a dedicated line, rather than a cellular configuration or a further kind of layout (i.e. job shop when variability of products is high and volumes per item low), considering frequent set-ups, such as those caused by batch switching, thus enabling the application of the Characteristic Curve [15] to manufacturing lines organized on a batch-production system. Even though no similar approaches are available for disassembly systems, their implementation is simply customizable for such environments. Thus, our study is located in a step following practical considerations executed by 
decision makers, which have guided the selection of a cellular configuration as a suitable layout for disassembly tasks. So, the detailed design of a cellular configuration follows by implementing published approaches.

Even though these assumptions seem appropriated, the underlying question is whether or not manufacturing and disassembly cells are different, from a cellular configuration point of view. If they are similar, solution approaches for the manufacturing cell formation problem may be efficiently applied to solve the disassembly cell formation problem. On the other hand, if they are different, a parallel theory must be built around this issue.

It is well known that reverse logistics systems are characterized by the uncertainties of quantity (variability of input items), quality (variability of conditions of similar items) and time of parts arrivals, and disassembly systems are no exception. Quality uncertainty means that a given product may arrive at the disassembly floor without several of its components. This could be due to the same product could be damaged during transport or handling and then supplied damaged to the disassembly system, that should check its status. So, the design of a cellular disassembly configuration should also follow the design of external and internal logistics, that could increment or decrease activities required in the production plan (i.e. for checking products integrity). An example of solutions that improve the internal and external logistics in WEEE (Waste of Electrical and Electronic Equipment) treatment networks is reported in Gamberini et al. [16].

This issue has been modeled as a relative frequency function $\left(f_{p j}\right)$ for which any operation $j$ is required on a product $p$, and consequently, the probability of any operation sequence $\pi_{p j j^{\prime \prime}}=f_{p j^{\prime}} \prod_{j<l<j^{\prime}} 1-f_{p l}$ is computed. The former is possible, because of the formulations being based on the concept of dedicated cells [17], which assigns operation types (instead of part types) to the cells, so that all operations of the same type are carried out in the same cell with the clear benefits that this brings in terms of learning effect, better quality, etc. If all disassembly operations were required for every product $\left(f_{p j}=1\right)$ we would face the typical case of assembly, which is a well-known 
situation. Quantity uncertainty means that product demand varies on a random basis, i.e. stochastic demand of products. This issue may be approached by stochastic programming formulations [18], robust optimization models [19], or fuzzy systems [20-21], among others.

It should be noted that the performance of a cellular manufacturing system, and particularly of a disassembly system, is highly dependent on the accuracy of the input data. It may rapidly deteriorate if demand and product quality changes. However, most of the current methods designed for the manufacturing cells formation problem have been developed for a single-period planning horizon. These models assume that problem data (e.g. product mix and demand) are constant for the entire planning horizon. We decided to use a robust approach because we elected to relax the assumptions of knowing the probability distribution of uncertain data. In the robust approach the product demand varies in a random manner. However, this variation can be described in a number of probabilistic scenarios with a given occurrence probability. The robustness of a layout is an indicator of its flexibility in handling demand variability. The aim of this approach is to obtain a cellular design in which the machines remain constant and do not move, only the flow of products changes once the demand has been observed.

The main aim of this research is to analyze the differences between cellular manufacturing and cellular disassembly systems. While doing so, this paper makes two relevant contributions. First it proposes a mathematical programming formulation for both the robust manufacturing cell formation problem (MCFP), and the robust disassembly cell formation problem (DCFP). Second, a new solution method is developed for both problems in order to obtain data in order to analyze the difference in the design of both types of production environment.

In the next section we propose robust optimization models for both problems to deal with demand uncertainty. In Section 3, we propose a metaheuristic approach to solve them. In Section 4, we describe the experimental design to evaluate the differences, identifying performance criteria to evaluate cellular configurations. Section 5 provides relevant conclusions. 


\section{Robust Optimization Models}

Robust optimization is a framework for modeling optimization problems that involve uncertainty. It has been applied to several areas of research and practice, such as production planning, machine programming, international supplies, and logistics. We refer to Cao and Chen [19] for an example in the manufacturing literature. The robust optimization methodology uses scenario-based approaches to deal with the uncertainty, where the scenarios correspond to the possible realizations of the uncertainty. The specification of a set of scenarios provides a way to incorporate different attitudes toward risk, relaxing the assumptions of knowing the probability distribution of uncertain data, thereby making a difference to stochastic programming. Defining the set of scenarios is a difficult task. It requires, for the decision maker, the dual mission of identifying the main factors causing uncertainty and describing the relationship between them. This process helps to reduce the number of scenarios, eliminating those that are unrealistic, and favoring its analysis.

The aim of the robust optimization approach in our cell formation problems is to design a cellular configuration that performs well across several potential realizations of uncertainty over a specified planning horizon. Since the definition of good performance is context-dependent, there exist several definitions of robustness [22-24]. Particularly relevant to our study is that provided by Mulvey et al. [22], which defines the concept of robustness as "finding a solution that minimizes the largest deviation from optimality".

Let $M$ and $P$ be, respectively, the set of machine/tools and the set of products that need to be dismantled or produced. Let $Q$ be the set of operations available for the respective process, and let

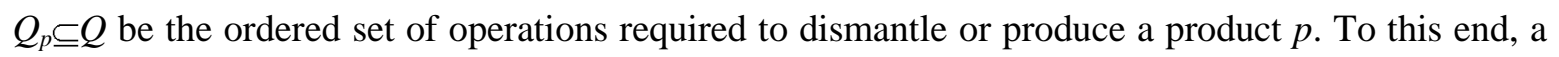
product $p$ and an operation $q$ are not independent, and each pair $\{p, q\}$ represents a task. Let $N=$ $\Sigma_{p \in P} \mathrm{card}\left(Q_{p}\right)$ be the total number of tasks, which have to be grouped into a subset of cells. We assume that the product demand (i.e. arrival of products to the disassembly floor or the orders of new products to be fulfilled by manufacturing, respectively) is not deterministic but can be described by several discrete scenarios with probabilities of their occurrences. For each product, the sequence in which the 
operations need to be performed is known. Two cost elements are considered: machine/tools acquisition (i.e. amortization) costs and material handling costs. Assuming that cell sizes are not large and that inter-cell distances are small, intercellular transportation costs are considered as a surrogate for material handling costs. The notation used in the mathematical formulation is described in Table 1.

\section{---- TABLE 1 HERE ----}

To formulate a robust optimization model [22], we assume that the decision variables related to the formation of cells $\left(y_{c}\right)$ and the decision variables related to the number of machines allotted to cells $\left(z_{m c}\right)$ are the design variables valid for all scenarios, and we let the decision to allocate the $j$-th operation of product $p$ to cell $c\left(x^{\omega}{ }_{p j c}\right)$ form the control variables, which can be adjusted once the demand is observed. Thus, the cells are formed and the machines are assigned to them, and then decisions regarding task and flow patterns are made for each scenario realization. Consequently, we will have a variable number of tasks processed in each cell $c$, in relation to each scenario $\omega \in \Omega$. We introduce the error or recourse variable $\mathrm{em}^{\omega}{ }_{c}$ to indicate any workload surplus in the machines of cell $c$. We also introduce an extra parameter $\lambda$ that the decision maker can adjust to give varying importance to the function that penalizes infeasibility in machines' capacity constraints.

The robust optimization problem is to determine the best cellular configuration for a given set of potential scenarios by grouping machines and assigning them to cells, so that total costs are minimized, while allowing tasks' and flow patterns' reconfiguration for each scenario realization. The mathematical model for the robust MCFP is given by:

$$
\text { Minimize } T C_{M F G}=\sum_{m \in M} \sum_{c \in C} \beta_{m} \cdot z_{m c}+\sum_{\omega \in \Omega} \rho_{\omega} \cdot \xi_{\omega}+\sum_{\omega \in \Omega} \sum_{c \in C} \rho_{\omega} \cdot \lambda \cdot e m_{c}^{\omega}
$$

subject to:

$$
\begin{array}{ll}
\sum_{c \in C} x_{p j c}^{\omega}=1 & \forall p \in P, j \in Q_{P}, \omega \in \Omega \\
L \cdot y_{c} \leq \sum_{p \in P} \sum_{j \in Q_{P}} x_{p j c}^{\omega} \leq U \cdot y_{c} & \forall c \in C, \omega \in \Omega
\end{array}
$$




$$
\begin{array}{ll}
\sum_{p \in P} \sum_{j: m \in O_{p j}} D_{p}^{\omega} \cdot \theta_{p j} \cdot x_{p j c}^{\omega}-e m_{c}^{\omega} \leq H_{m} \cdot z_{m c} & \forall m \in M, c \in C, \omega \in \Omega \\
x_{p j c}^{\omega} \in\{0,1\} & \forall p \in P, j \in Q_{p}, c \in C, \omega \in \Omega \\
y_{c} \in\{0,1\} & \forall c \in C \\
z_{m c} \geq 0 \text { and integer } & \forall m \in M, c \in C \\
e m_{c}^{\omega} \geq 0 & \forall c \in C, \omega \in \Omega
\end{array}
$$

The objective function in Equation (1) minimizes the total cost. The first term represents the machine amortization cost. The second term measures the solution-robustness by minimizing the expected value of the intercellular material handling cost, where:

$$
\xi_{\omega}=\sum_{p \in P} \sum_{j \in Q_{p}} \sum_{j<j^{\prime}} \sum_{c \in C} \alpha_{p} \cdot D_{p}^{\omega} \cdot x_{p j c}^{\omega} \cdot \mathbf{1}-x_{p j^{\prime} c}^{\omega}
$$

is the intercellular material handling cost for scenario $\omega$. This is a nonlinear function which can be easily linearized according to [13]. Finally, the third term measures the model-robustness by minimizing the expected value of the associated error.

The constraint in Equation (2) sets a feasibility condition. It ensures that in scenario $\omega$, the $j$-th operation of product $p$, will be assigned to one, and only one, cell. The constraints in Equations (3) provide the lower and upper bound conditions of cells. Capacity conditions are given by Equation (4). This guarantees that machine capacities are not exceeded, while ensuring that all product demands are met. The parameter $\theta_{p j}$ implies that during the time required by the $j$-th operation of product $p$, the machine/tool type $m$ cannot be used in any other operation; all that time corresponds to the machine type $m$, regardless of whether the use of other machines was also considered in the total duration time of the operation. Finally, Equations $(5) \sim(8)$ deal with the nature of the decision variables. It should be noted that although the recourse variables are declared as continuous, the formulation forces them to take integer values.

Now, in order to formulate the robust DCFP, the following equation must be replaced in the objective function: 


$$
\xi_{\omega}=\sum_{p \in P} \sum_{j \in Q_{p}} \sum_{j<j^{\prime}} \sum_{c \in C} \alpha_{p} \cdot D_{p}^{\omega} \cdot f_{p j} \cdot \pi_{p j j^{\prime}} \cdot x_{p j c}^{\omega} \cdot \mathbf{1}-x_{p j^{\prime} c}^{\omega}
$$

As well as the constraint in equation (4):

$$
\sum_{p \subseteq P} \sum_{j: m \subseteq O_{p j}} D_{p}^{\omega} \cdot \theta_{p j} \cdot f_{p j} \cdot x_{p j c}^{\omega}-e m_{c}^{\omega} \leq H_{m} \cdot z_{m c} \quad \forall m \in M, c \in C, \omega \in \Omega
$$

The CFP is essentially an NP-hard problem, as it is a variation of the fixed charge problem with additional decision variables [25]. The robust optimization formulations proposed here, to capture the demand uncertainty in the manufacturing and disassembly systems, result in more complicated problems than the classical cell formation problem, with additional decision variables associated with a set of potential scenarios. Therefore it is usually inefficient to use an exact algorithm to solve the CFP for real-life instances. Actually, to solve practical problems, feasible integer solutions are obtained after a reasonable computational time in a commercial MIP solver.

\section{Solution Approach}

In order to analyze the results obtained with the above models, and given their complexity, a Tabu Search algorithm was used. Tabu Search is a traditional metaheuristic which has been proposed for solving different kinds of cell formation problems [4]. Our implementation is based on the design of Adenso-Díaz and Lozano [17], which is also similar to those proposed previously and later for a wide variety of problems of cellular design [13, 25-28], including robust designs [19]. The fact that this Tabu Search algorithm has been effective in different cell formation approaches gives us confidence in its reliability, and although alternative metaheuristic approaches may be developed in the future, we opted for adapting this algorithm to the problem at hand, since our first aim is to obtain data to analyze the difference in the design of both types of production environments but not to develop new approaches for MCFP. The remainder of this section describes the modifications required in the Tabu Search algorithm to solve our particular problems.

Specifically, a solution with $N$ tasks will be represented by an array $\hat{x}_{\omega}=\left\langle x_{\omega}(1), x_{\omega}(2), \ldots, x_{\omega}(N)\right\rangle$,

for each scenario, in which the cardinality constraints are met. Each position of $\hat{x}_{\omega}$ indicates the cell $c$ 
to which the $i$-th task has been assigned in scenario $\omega\left(x_{\omega}(i)=c\right)$. To comply with cardinality constraints a feasible solution must satisfy that $L \leq W_{\omega}(c) \leq U$ for all $c$ and for all $\omega$, where $W_{\omega}(c)$ is the number of tasks assigned to cell $c$ in scenario $\omega$. See Fig. 1. For a given solution, the number of machines of each type required in each cell for scenario $\omega\left(z_{m c}^{\omega}\right)$ can be obtained using the constraints in Equations (4) and (4'), respectively, for each production environment, as in Equation (10):

$$
z_{m c}^{\omega}=\left\lceil\frac{1}{H_{m}} \sum_{p \in P} \sum_{i=l}^{u p_{p}^{-}} D_{p}^{\omega} \cdot \theta_{i}\right\rceil
$$

\section{---- FIGURE 1 HERE ----}

Thus, the number of machines required in the robust solution can be computed as $z_{m c}=\left\lceil\sum_{\omega \in \Omega} \rho_{\omega} \cdot z_{m c}^{\omega}\right\rceil$, while the workload surplus in the machines of cell $c$ for scenario $\omega$ is computed as in Equation (11):

$$
\operatorname{em}_{c}^{\omega}=\max \left\{0, \sum_{m \in M}\left(\sum_{p \in P} \sum_{i=l}^{u \varphi_{p} \hat{x}_{\omega}} D_{p}^{\omega} \cdot \theta_{i}-z_{m c} \cdot H_{m}\right)\right\}
$$

Finally, it is easy to compute the total cost associated with solution $x$ as follows:

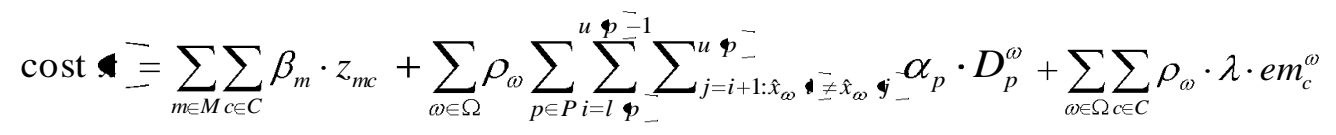

From the initial solution onwards, a Tabu Search commences by exhaustively exploring the neighborhoods with three types of movement: [swap] exchange of cells between two tasks, $i, j$, that are carried out in different cells $\left(x_{\omega}(i) \leftrightarrow x_{\omega}(j), x_{\omega}(i) \neq x_{\omega}(j)\right)$; [insert $]$ add an operation $i$ to a different cell from the one presently in $\left(x_{\omega}(i) \leftarrow c^{\prime}, c^{\prime} \neq x_{\omega}(i)\right)$; [union] merge all the operations of one cell with those of the other (thus reducing the total number of cells). These moves are typically used in the design of metaheuristic algorithms for the CFP [13, 27, 29-30]. With the aim of being able to diversify the search after a certain number of iterations without improvement $(\gamma)$, the $[$ split $]$ move will divide the cell with the largest number of tasks into two (thus incrementing the total number of cells). The search ends after max_iter iterations without improvement. Two short-term memory structures 
are considered to keep track of moves that remain tabu during a specified time horizon (tabu tenure). The swap and insert operations are associated with these tabu mechanisms, where the respective tabu tenures are $\delta$ and $\varphi$ iterations respectively. The aspiration criterion used was the standard aspiration criterion used in most Tabu Search applications, which states that a tabu move is accepted if the solution it produces is better than the best solution found to date.

\section{Computational Experiments}

The above algorithm was coded in $\mathrm{C}$ using the Microsoft Visual $\mathrm{C}++6.0$ programming environment via the Windows 7 operating system. All experiments were conducted on an HP Z800 Workstation with a processor Intel Xeon X5647 operating at $2.93 \mathrm{GHz}$ and $12.00 \mathrm{~GB}$ RAM. Preliminary numerical tests were used to set the values of the required parameters for the Tabu Search algorithm. The diversification procedure is applied after $\gamma=20$ iterations without improvement. On the other hand, the stopping criterion is specified in terms of max_iter $=90$ successive iterations without improvement in the objective function. Finally, the tabu tenures were fixed at $\delta=10$ and $\varphi=$ 15 iterations respectively.

\subsection{Experimental Framework}

This section describes the experimental framework used to analyze the differences between cellular manufacturing and cellular disassembly systems. A full factorial design study is proposed in order to appraise the significance of controllable factors in cellular designs. What is at issue here is to see what happens with the solutions of each production environment (manufacturing and disassembly) when varying factors, in order to analyze the differences and similarities between both. To do so, diverse instances of different sizes are required to carry out the computational tests, considering a set of factors for which there is a proved conjecture to affect cellular designs. The following factors were considered:

- F1- number of products: the number and demand of products are two important factors which justify a cellular layout $[17,31]$. Therefore the former factor is considered with three levels $\left\{\mathrm{F}_{11}-10\right.$ products, $\mathrm{F}_{12}-20$ products, $\mathrm{F}_{13}-50$ products $\}$ that reflect different problem sizes. 
- F2 - demand variability between scenarios: when dealing with robust cellular designs, defining the number of scenarios is an issue [24] which affects the problem size. For experimental purposes we keep the number of scenarios fixed on 4 with a $25 \%$ of occurrence probability each, as we are not interested in analyzing the solution's quality regarding the problem complexity, but in assessing the significance of controllable factors in cellular designs. In a robust design, the demand variability between scenarios is a factor which undoubtedly affects the decisions made. Therefore, two levels are considered for this factor $\left\{F_{21}-10 \%\right.$ of demand variability between scenarios, $\mathrm{F}_{22}-40 \%$ of demand variability between scenarios $\}$, a $10 \%$ of demand variability between scenarios implies that in every scenario the demand remains almost constant. On the other hand a $40 \%$ of demand variability implies a high degree of uncertainty in every scenario.

- F3 - material handling cost: one of the well-known benefits of cellular layouts is a reduction in material handling and lead times [1, 32-33]. Most of the cellular manufacturing models published in the literature consider the intercellular movement cost as a surrogate for material handling cost. Usually, mathematical models aim to create a trade-off between machine amortization cost and material handling costs, while minimizing total costs. However, to keep the study focused, only the material handling costs are considered as a factor. $\left\{F_{31}-U \sim(0.5-1.0)\right.$, $\left.\mathrm{F}_{32}-U \sim(2.0-2.5)\right\}$

- F4- uncertainty in quality of arriving products: a relative frequency function which is used to measure the probability that a disassembly operation is required for a given product, which is characteristic of disassembly systems $[12,34]$. In order to generate the instances, for each task (product-operation) a random number (rand) is generated; if rand $<0.4$, then the relative frequency function $f_{p j}=1$, else $f_{p j}=U \sim(0.15-0.30)$ when $\mathrm{F}_{41}$, or $f_{p j}=U \sim(0.50-0.75)$ when $\mathrm{F}_{42}$, and $f_{p j}=U \sim(0.85-1.00)$ when $\mathrm{F}_{43}$. Scenario $\mathrm{F}_{41}$ means that products arriving at the disassembly floor present a high degree of damage (many components are missing); on the other hand $\mathrm{F}_{43}$ represents a scenario where almost all products arrive at the disassembly floor with all their components; the last scenario resembles assembly environments. 
The advantages of using cellular manufacturing configurations for regular production are well known. For example, in some cases simply rearranging existing machines into a cellular configuration is sufficient to provide significant benefits such as reductions in: throughput time, in-process inventory, scrap, labor, set-up times, material handling costs, and floor space. Also higher machine utilization rates, better production efficiency and quality as well as market response time may be achieved by using cellular configurations [35-37]. On the other hand, cellular configurations could cause loss in the flexibility of the system because machines will now be dedicated to a few part-families instead of being available for more general purposes [38]. In our experiments, four dependent variables were considered to assess the performance of a cellular configuration for each production environment:

- the total cost,

- the average ratio of cell utilization,

- the maximum number of intercellular moves, and

- the average percentage of machine utilization.

The former is used as a criterion to measure the quality of the solution, while the remaining three criteria are used to measure the design of the solution. The average ratio of cell size utilization regarding the maximum number of operations assigned is used as a surrogate for cell size. It should be noted that the size of the cells was not significant because solutions with the minimum number of cells are preferred, and the algorithm always provides solutions with this bound.

In order to avoid any bias in the design of the instances, three diverse instances of different sizes were taken from the literature, where the operation sequences and machine types for a set of products are described; these are presented in Table 2. Each instance considers a different number of products and operations. However, as the proposed models do not coincide exactly with any model in the literature, not even with respect to the input data considered, instead of generating random instances we opted for using the problems reported in the literature as a data base, completing these if 
necessary. Thus, for each one of these instances, the missing data were fixed semi-randomly, i.e. making sure that the order of magnitude of the data in question was not unreasonable. The full data employed are available on request from the authors.

\section{---- TABLE 2 HERE ----}

For each instance type, where the number of products is fixed (F1), we define a class as a combination with the factors F2 and F3. In this sense, the class $\prec 2,2,1>$ represents an instance with 20 products, a high variability of demand between scenarios, and low material handling costs. For each instance class, three replicates were generated. In order to analyze the differences between manufacturing and disassembly environments, the instances generated for the manufacturing environment were taken as a base to generate the instances for the disassembly environment, complementing them with factor F4. As a result 144 paired instances $\left(3 \times 3 \times 2^{2}=36\right.$ for manufacturing, and 108 for disassembly) were solved in order to derive conclusions. The computational times were very low in all cases.

\subsection{Analysis of Factors in Cellular Designs}

A first experiment was designed to consider the main and interactive effects within to screen the significant effects and interactions via the Analysis of Variance (ANOVA) for each dependent variable in each production environment (manufacturing and disassembly). It is important to note that when using ANOVA, three main assumptions must be checked: normality, homogeneity of variance, and independence of residuals. We did those checks for every experiment, and found no basis for questioning their validity. In the remaining sections we discuss and analyze the differences between cellular manufacturing and cellular disassembly systems for each one of the four response variables.

The box plot in Fig. 2 shows that for the manufacturing case, the total cost behaves differently as the number of products changes. The ANOVA in Table 3a shows that the number of products (F1), as well as the material handling cost (F3) factors, were primarily responsible for the total cost

configuration in the manufacturing systems. The number of products, the material handling cost, and 
the interaction between both factors, contributed to a total of about $99 \%$ of the variances in the total cost in the manufacturing case. A post hoc Tukey test (Table 3b) shows the presence of significant differences in the number of products and material handling cost levels, relative to the total cost. This is not surprising, given that F3 is the movement cost. However, for demand variability (F2), no significant differences between $10 \%$ and $40 \%$ were found.

---- FIGURE 2 HERE ----

---- TABLE 3 HERE ----

The box plot in Fig. 3 shows that for the disassembly case, as in the manufacturing case, the total costs behave differently as the number of products changes, but the product frequency also has an impact on this response variable. The ANOVA shows that for the disassembly case, the number of products $(\mathrm{F} 1)$, the material handling cost $(\mathrm{F} 3)$, and the product frequency (F4) factors in the disassembly systems were primarily responsible for the total cost configuration. The number of products $(\mathrm{F} 1)$, the material handling cost $(\mathrm{F} 3)$, the interaction between both, the product frequency (F4), and the interaction between number of products and product frequency contributed to a total of about $90 \%$ of the variances in the total cost. On average, the total cost in the manufacturing case is $33.15 \%$ higher than in the disassembly case. However the 2-sample t-test reveals that there is insufficient evidence to conclude that the means of total costs in both production environments differ at the 0.05 level of significance.

\section{---- FIGURE 3 HERE ----}

---- TABLE 4 HERE ----

Similar analyses were performed for the cell utilization, number of movements, and machine utilization, revealing the following findings (all results are significant at 0.05):

(a) For the average ratio of cell utilization, the effects of the number of products (F1), the material handling costs (F3) and the interaction between both, accounted for $56.44 \%$ of the variances in the disassembly case (see Table 5), while in the manufacturing case the number of products was primarily responsible for the cell utilization, contributing to a total 
of about $39 \%$ of the variances. It should be noted that not only F4 is not particularly relevant, but also the difference in the average ratio of cell utilization for both production environments is lower than $1 \%$. This could be explained because cell utilization accounts for the number of operations assigned to a cell, and in both cases solutions with the smallest number of cells are preferred. So there is not enough statistical evidence to conclude that on average the cells in one production environment are smaller than in the other.

---- TABLE 5 HERE ----

(b) The Number of Intercellular Movements, the effects of number of products (F1), material handling cost (F3), product frequency (F4), and the interaction between them accounted for $96.19 \%$ of the variances in the disassembly case (see Table 6a), while in the manufacturing case the number of products (F1) and the material handling cost (F3) were primarily responsible for the number of movements, contributing to a total of about $99.26 \%$ of the variances (see Table 6b). On average, there are $20 \%$ more movements in disassembly than in manufacturing.

\section{---- TABLE 6 HERE ----}

(c) For Machine Utilization, the effects of number of products (F1) and the product frequency (F4) accounted for $68.67 \%$ of the variances in the disassembly case (see Table 7a), while in the manufacturing case the number of products (F1) and the demand variability between scenarios (F2) were primarily responsible for the machine utilization, contributing to a total of about $61.93 \%$ of the variances (see Table $7 \mathrm{~b}$ ). The average percentage of machine utilization is $10.71 \%$ higher in the disassembly case than in the manufacturing case. There are fewer machines but their utilization rate is higher.

---- TABLE 7 HERE ----

\subsection{Comparing Cellular Designs in Manufacturing and Disassembly Environments}

The former analyses show that F4 is a significant factor for every response variable in the disassembly case. Therefore, a second experiment considers the main and interactive effects within 
factor to screen the significant effects and interactions of each level of F4 in a disaggregated form, via the multivariate analysis of variance (MANOVA). A deeper analysis was conducted on paired samples for the manufacturing case and three scenarios of the disassembly case. Each scenario of a disassembly case differs from the others (including the manufacturing case) in the frequency of the required operations (F4). To do so, factors F1 to F3 were considered, and an additional factor (F5) was introduced to identify the proper production environment as follows: $F_{51}$-manfuacturing, $\mathrm{F}_{52}$-Dissasembly with low product frequency, $\mathrm{F}_{53}$-Dissasembly with moderate product frequency, and $\mathrm{F}_{54}$-Dissasembly with high product frequency (closely similar to the manufacturing case).

We decide to use MANOVA in order to minimize the probability of making one or more type I errors (i.e. to conclude that a difference exists when one does not) for the entire set of comparisons. The MANOVA is used to determine whether there are any differences between independent groups on more than one dependent variable. Therefore, we use a MANOVA to understand whether there were differences in manufacturing and disassembly environments based on three different performance indicators (total cost, cell size, and machine utilization). With MANOVA we take advantage of the data covariance structure to simultaneously test the equality of means from different responses. MANOVA requires checking nine assumptions: (1) the dependent variables should be measured at the interval or ratio level (i.e. they are continuous); (2) the independent variable should consist of two or more categorical, independent groups (manufacturing and disassembly with different quality product uncertainty); (3) independence of observations, which means that there is no relationship between the observations in each group or between the groups themselves; (4) an adequate sample size; (5) no univariate or multivariate outliers; (6) multivariate normality; (7) a linear relationship between each pair of dependent variables for each group of the independent variable; (8) a homogeneity of variance-covariance matrices, and (9) no multicollinearity.

With the aim of examining individually each one of the three response variables, an ANOVA was performed for each variable within this experiment. Each analysis of variance evaluates the effects of the number of products $(\mathrm{F} 1)$, demand variability between scenarios $(\mathrm{F} 2)$, the material handling costs 
(F3), the production environment (F5), and their interaction at two levels of each factor on each response variable. For the total cost, the results indicate that: the number of products, the material handling costs and the production environment (manufacturing and each disassembly process) are significant ( $\mathrm{p}$-value $=0.000$ ). In other words, a significant amount of variation in the total cost is associated with variation in these three factors. The interaction between the number of products and material handling cost (0.000), as well as the interaction between the material handling cost and the production environment (0.039) are also significant; therefore we cannot consider the effects of both factors separately. The adjusted $R^{2}=96.30 \%$ indicates the adequacy of the model. For the size of cells $\left(R^{2}=72.65 \%\right)$, the results indicate that factors $\mathrm{F} 1$ and $\mathrm{F} 3$, as well as the two-level interaction between them, are significant; however, the production environment (F5) is not a significant factor, so we can consider their effects separately. This implies that the production environment does not affect the size of the cells. Finally, the ANOVA results for the machine utilization $\left(R^{2}=77.04 \%\right)$ indicate that the number of products, the demand variability and the production environment affect the percentage of machine utilization. However, the interaction between them is not significant.

We examine the variability, error, and partial correlations matrices to assess the performance of the MANOVA. To appraise how the response variables are related, a partial correlation between them is computed. The partial correlation of 0.4854 between total cost and machine utilization is moderated; however, the partial correlation of -0.2444 between total cost and cell size is not large, and the partial correlation of -0.0538 between cell size and machine utilization is small. Because the correlation structure is not weak enough, the MANOVA is adequate.

\section{---- TABLE 8 HERE ----}

We use the Wilks' test to judge where there is significant evidence for model effects. In Table 8, the p-values for the F1 to F5 factors show that different levels of each factor affect the responses differently; also, there is significant evidence for interaction between F1 and the remaining factors, but there is no significant evidence for interaction between F2-F3 and F2-F5 at $\alpha$ level 0.05 . The second column in Table 8 shows the relative contribution of each factor over each response variable. 
The Eigen analysis was used to assess how the response means differ among the levels of the different factors. From the results of the Eigen analysis, it is evident that the percentage of machine utilization means having larger differences among the factor levels for number of products (F1), demand variability (F2), handling cost (F3) and production environment (F5), and in some interactions between them. But at the two factor interaction level between F1and F2, the size of the cells' means have the largest differences. The total costs' means have the smallest differences at a two factor interaction on every factor.

From the previous analysis, we conclude that: (a) the number of products is statistically significant for the three response variables, and is in most of the cases the principal response of the variance; (b) the production environment is also statistically significant for the three response variables, but it mainly affects the machine utilization rate; (c) the two factor interactions of the production environment with the number of products, and with the handling costs, are statistically significant; (d) there is enough statistical evidence to conclude that the production environment affects the three performance indicators of a cellular system; in particular, the machine utilization rate and size of the cells are the performance indicators that have the greatest impact.

The MANOVA reveals that at least two groups were different. A post-hoc test was conducted to determine which of these groups differ from the others (see Fig. 4).

\section{---- FIGURE 4 HERE ----}

\section{Conclusions}

The differences between cellular manufacturing and cellular disassembly systems have been studied in this paper. A mathematical programming formulation for the robust manufacturing and disassembly cell formation problems has been proposed, and a solution method based on the Tabu Search schema is adapted for both problems, and was used for the comparison analysis, since our first aim is to obtain data to analyze the difference in the design of both types of production environment and not to develop new approaches to the cell formation problem. 
Four dependent variables were considered in the experiments: total cost, size of the cells, number of intercellular moves, and machine utilization. A design of experiments approach based on the analysis of variance and multivariate analysis of variance is used in order to appraise the significance of controllable factors in cellular designs. The first set of experiments considers the main and interactive effects within the factors to screen the significant effects and interactions, via the Analysis of Variance for each dependent variable, on each production environment (manufacturing and disassembly). Results show that in the disassembly case, the uncertainty in the quality of arriving products is a significant factor for every response variable. Therefore, a second experiment screens the significant effects and interactions of each level of uncertainty in the quality of arriving products in a disaggregated form, via the multivariate analysis of variance with paired samples for the manufacturing case and three scenarios of the disassembly case.

Results also show that the production environment affects the three performance indicators of a cellular system; in particular the machine utilization rate and size of the cells are the performance indicators that have the greatest impact. This means that disassembly and manufacturing systems differ. From a managerial point of view, it appears that both systems will behave differently as the uncertainty in the quality of arriving products increases. For example, there are production systems where the assembly and disassembly operations are performed on the same production floor, as in the manufacturing of electric engines. In this case all engines contain all their parts, as they have not gone through a cannibalism phase. On the other hand, when the products arrive from outside the facility many components are missing, therefore disassembly cells tend to be smaller and the disassembly operation is cheaper.

\section{Acknowledgements}

The research activity of JLGV has been partially funded by Tecnológico de Monterrey - Research Group in Industrial Engineering and Numerical Methods 0822B01006 and by the Mexican National Council for Science and Technology (CONACyT) through grant SEP-CONACyT CB-2011-01-166397. JMO acknowledges the support of Universidad Autónoma de Tamaulipas 
through research fund PFI2014-34. BA-D is granted by the Spanish Ministry of Science; grant DPI2013-41469-P and the European Regional Development Fund (ERDF).

\section{References}

[1]. Wemmerlöv U, Hyer N (1989) Cellular manufacturing in the US industry: A survey of users. Int J Prod Res 27(9): 1511-1530

[2]. Singh N (1993) Design of cellular manufacturing systems: An invited review. Eur J Oper Res 69(3): 281-511

[3]. Selim HM, Askin RG, Vakharia AJ (1998) Cell formation in group technology: review, evaluation and directions for future research. Comput Ind Eng 34(1): 3-20

[4]. Papaioannou G, Wilson JM (2010) The evolution of cell formation problem methodologies based on recent studies (1997-2008): Review and directions for future research. Eur J Oper Res 206(3): 509-521

[5]. Adenso-Díaz B, Lozano S, Andrés C, García JM (2006) Disassembly cells design. Proceedings of the Group Technology/Cellular Manufacturing Conference, University of Gröningen, The Netherlands, 443-450

[6]. Mar-Ortiz J, Lozano S, Adenso-Díaz B, González B, González-Velarde JL (2009) Heuristic Procedure for the Disassembly Cells Formation Problem. Proceedings of the 5th International Conference on Intelligent Manufacturing and Logistics Systems, Kitakyushu, Japan

[7]. Kopacek B, Kopacek P (1999) Intelligent disassembly of electronic equipment. Annu Rev Control 23: $165-170$

[8]. Teunter RH (2006) Determining optimal disassembly and recovery strategies. Omega-Int J Manage S 34(6): 533-537

[9]. Ilgin MA, Gupta SM (2010) Comparison of economic benefits of sensor embedded products and conventional products in a multi-product disassembly line. Comput Ind Eng 59(4): $748-763$

[10]. Tang Y, Zhou MC, Zussman E, Caudill R (2002) Disassembly modelling, planning, and application. J Manuf Syst 21(3): 200-217

[11]. González B, Adenso-Díaz B (2006) A scatter search approach to the optimum disassembly sequence problem. Comput Oper Res 33(6): 1776-1793

[12]. Andrés C, Lozano S, Adenso-Díaz B (2007) Disassembly sequence planning in a disassembly cell context. Robot CIM-INT Manuf 23(6): 690-695

[13]. Mar-Ortiz J, González-Velarde JL, Adenso-Díaz B (2014) A VNS algorithm for a disassembly cell formation problem with demand variability. Eur J Ind Eng 8(1): 22-49 
[14]. Deming DD (1959) When to shift to straight-line production. Harvard Bus Rev 38: 62-68

[15]. Gamberi M, Gamberini R, Manzini R, Regattieri A (2008) An analytical model to evaluating the implementation of a batch-production-oriented line. Int J Prod Econ 111(2): 729-740

[16]. Gamberini R, Gebennini E, Rimini B (2009) An innovative container for WEEE collection and transport: details and effects following the adoption. Waste Manage 29(11): 2846-2858

[17]. Adenso-Díaz B, Lozano S (2008) A model for the design of dedicated manufacturing cells. Int J Prod Res 46(2): 301-319

[18]. Tavakkoli-Moghaddam R, Javadian N, Javadi B, Safaei N (2007) Design of a facility layout problem in cellular manufacturing systems with stochastic demands. Appl Math Comput 184(2): 721-728

[19]. Cao D, Chen M (2005) A robust cell formation approach for varying product demands. Int J Prod Res 43(8): 1587-1605

[20]. Safaei N, Saidi-Mehrabad M, Babakhani M (2007) Designing cellular manufacturing systems under dynamic and uncertain conditions. J Intell Manuf 18: 383-399

[21]. Papaioannou G, Wilson JM (2009) Fuzzy extensions to Integer Programming models of cell-formation problems in machine scheduling. Ann Oper Res 166(1): 163-181

[22]. Mulvey JM, Vanderbei RJ, Zenios SA (1995) Robust optimization of large-scale systems. Oper Res 43(2): 264-281

[23]. Kouvelis P, Yu G (1997) Robust discrete optimization and its applications. Kluwer Academic Publishers, Norwell, MA

[24]. Ben-Tal A, El-Ghaoui L, Nemirovski A (2009) Robust Optimization. Princeton University Press

[25]. Cao D, Chen M (2004) Using penalty function and Tabu search to solve cell formation problems with fixed cell cost. Comput Oper Res 31(1): 21-31

[26]. Lozano S, Adenso-Díaz B, Eguia I, Onieva L (1999) A one-step Tabu search algorithm for manufacturing cell design. J Oper Res Soc 50(5): 509-516

[27]. Schaller J (2005) Tabu search procedures for the cell formation problem with intra-cell transfer costs as a function of cell size. Comput Ind Eng 49: 449-462

[28]. Chung SH, Wu TH, Chang CC (2011) An efficient tabu search algorithm to the cell formation problem with alternative routings and machine reliability considerations. Comput Ind Eng 60(1): 7-15

[29]. Aljaber N, Baek W, Chen CL (1997) A tabu search approach to the cell formation problem. Comput Ind Eng 32(1): 169-185 
[30]. Spiliopoulos K, Sofianopoulou S (2008) An efficient ant colony optimization system for the manufacturing cells formation problem. Int J Adv Manuf Technol 36(5-6): 589-597

[31]. Askin RG, Standridge CR (1993) Modeling and analysis of manufacturing systems. John Wiley and Sons

[32]. Askin RG, Subramanian PS (1987) A cost-based heuristic for group technology configuration. Int J Prod Res 25(1): 101-113

[33]. Askin RG, Selim HM, Vakharia AJ (1997) A methodology for designing flexible cellular manufacturing systems. IIE Trans 29(7): 599-610

[34]. Brennan L, Gupta SM, Taleb KN (1994) Operations Planning Issues in an Assembly/Disassembly Environment. Int J Oper Prod Man 14(9): 57-67

[35]. Levasseur GA, Helms MM, Zink AA (1995) A conversion from a functional layout to a cellular manufacturing layout at Steward, Inc. Prod Inv Manag J 36: 37-42

[36]. Safaei N, Tavakkoli-Moghaddam R (2009) Integrated multi-period cell formation and subcontracting production planning in dynamic cellular manufacturing systems. Int J Prod Econ 120(2): 301-314

[37]. Kia R, Baboli A, Javadian N, Tavakkoli-Moghaddam R, Kazemi M, Khorrami J (2012) Solving a group layout design model of a dynamic cellular manufacturing system with alternative process routings, lot splitting and flexible reconfiguration by simulated annealing. Comput Oper Res 39(11): 2642-2658

[38]. Balakrishnan J, Jog PD (1995) Manufacturing cell formation using similarity coefficients and a parallel genetic TSP algorithm: Formulation and comparison. Math Comput Model 21(12): $61-73$

[39]. George AP, Rajendran C, Ghosh S (2003) An analytical-iterative clustering algorithm for cell formation in cellular manufacturing systems with ordinal-level and ratio-level data. Int J Adv Manuf Technol 22(1-2): 125-133 


\section{LIST OF TABLES}

Table 1. Notation used in the mathematical model.

\begin{tabular}{|c|c|}
\hline \multicolumn{2}{|r|}{ Sets } \\
\hline$P$ & Set of product types. \\
\hline$Q$ & Set of disassembly/manufacturing operations. \\
\hline$Q_{p}$ & $Q_{p} \subseteq Q$, subset of operations required by product $p \in P$. \\
\hline$C$ & Set of cells to be formed. \\
\hline$M$ & Set of machines. \\
\hline$\Omega$ & Set of scenarios. \\
\hline \multicolumn{2}{|r|}{ Parameters } \\
\hline$D_{p}^{\omega}$ & Demand for product type $p$ in scenario $\omega$. \\
\hline$\alpha_{p}$ & $\begin{array}{l}\text { Cost to move one unit of product } p \text { from one cell to another. } \\
\text { (intercellular material handling cost). }\end{array}$ \\
\hline$\rho_{\omega}$ & Occurrence probability in scenario $\omega$. \\
\hline$O_{p j}$ & Set of machines required by the $j$-th operation of product $p$. \\
\hline$\theta_{p j}$ & Duration of the $j$-th operation on product $p$. \\
\hline$f_{p i}$ & Relative frequency of the $j$-th operation of product $p$. It should be noted that $0 \leq f_{p j} \leq 1$. \\
\hline$\pi_{p j j}$ & Probability that the $j$ '-th of product $p$ be required immediately after the $j$-th operation $(j$ \\
\hline & $\left.<j^{\prime}\right)$, where: $\pi_{p j j^{\prime}}=f_{p j^{\prime}} \prod_{j<l<j^{\prime}} \mathbf{1}-f_{p l}$. \\
\hline$H_{m}$ & Capacity of machine of type $m$ in time units. \\
\hline$\beta_{m}$ & Amortization cost of machine of type $m$. \\
\hline$U$ & Maximum number of tasks allowed per cell. \\
\hline$L$ & Minimum number of tasks allowed per cell. \\
\hline$\lambda$ & $\begin{array}{l}\text { Cost which penalizes the infeasibility in the machine's capacity constraint (adjusted by } \\
\text { the decision maker). }\end{array}$ \\
\hline \multicolumn{2}{|r|}{ Binary decision variables } \\
\hline $\begin{array}{c}x_{p j c}^{\omega} \\
y_{c}\end{array}$ & $\begin{array}{l}1 \text { if the } j \text {-th operation of product } p \text { is assigned to cell } c \text { in scenario } \omega \text {, and } 0 \text { otherwise. } \\
1 \text { cell } c \text { is formed, and } 0 \text { otherwise. }\end{array}$ \\
\hline \multicolumn{2}{|r|}{ Integer decision variables } \\
\hline$z_{m c}$ & Number of machines of type $m$ installed in cell $c$. \\
\hline \multicolumn{2}{|r|}{ Continuous decision variables (recourse variables) } \\
\hline$e m^{\omega}{ }_{c}$ & Workload surplus in the machines of cell $c$ for scenario $\omega$. \\
\hline
\end{tabular}


Table 2. Set of instances considered.

\begin{tabular}{clccc}
\hline $\begin{array}{c}\text { Instance } \\
\text { Type }\end{array}$ & Base Reference & $|P|$ & $|Q|(N)(G)$ & $|M|$ \\
\hline Set01 & Cao and Chen (2005) & 10 & $6(25)(3)$ & 6 \\
Set02 & George, Rajendran and Ghosh (2003) & 20 & $8(61)(5)$ & 8 \\
Set03 & Spiliopoulos and Sofianopoulou (2008) & 50 & $30(154)(6)$ & 30 \\
\hline
\end{tabular}

Note: $|P|$ is the number of products in the instance, $|Q|$ the total number of operations, the total number of tasks $\left(N=\Sigma_{p}\left|Q_{p}\right|\right), G=\max _{p}\left\{\left|Q_{p}\right|\right\}$ is the maximum number of operations required by any product, $|M|$ is the number of types of machine. 
Table 3.ANOVA and post hoc Tukey test for relevant factors in the manufacturing case (total cost).

\begin{tabular}{|c|c|c|c|c|c|c|}
\hline Source & df & Sum of squares & Mean Square & p-value & $\begin{array}{l}\text { Contri. } \\
(\%)\end{array}$ & $\begin{array}{c}\text { Cum. Contri } \\
(\%)\end{array}$ \\
\hline F1 & 2 & 18968064 & 9484032 & 0.000 & 90.247 & 90.24 \\
\hline $\mathrm{F} 1 * \mathrm{~F} 3$ & 2 & 1105131 & 552565 & 0.000 & 5.258 & 95.50 \\
\hline F3 & 1 & 792047 & 792047 & 0.000 & 3.768 & 99.27 \\
\hline $\mathrm{F} 1 * \mathrm{~F} 2$ & 2 & 8748 & 4374 & 0.467 & 0.042 & 99.31 \\
\hline $\mathrm{F} 2$ & 1 & 7954 & 7954 & 0.244 & 0.038 & 99.35 \\
\hline $\mathrm{F} 1 * \mathrm{~F} 2 * \mathrm{~F} 3$ & 2 & 1495 & 748 & 0.875 & 0.007 & 99.36 \\
\hline $\mathrm{F} 2 * \mathrm{~F} 3$ & 1 & 709 & 709 & 0.724 & 0.003 & 99.36 \\
\hline Error & 24 & 133734 & 5572 & & 0.636 & 100.00 \\
\hline Total & 35 & 21017882 & & & & \\
\hline
\end{tabular}

(a) ANOVA.

\begin{tabular}{lccrr}
\hline Factor & Levels compared & \multicolumn{1}{c}{ Diff } & signification \\
\hline F1 & 1 & 2 & 134266.3 & 0.0005 \\
& 1 & 3 & 1602544.3 & 0.0000 \\
& 2 & 3 & 1468278.0 & 0.0000 \\
F2 & 1 & 2 & 29727.67 & 0.2438 \\
F3 & 1 & 2 & 296656.8 & 0.0000 \\
\hline
\end{tabular}

(b) Post hoc Tukey test. 
Table 4.ANOVA for Total Cost in the disassembly case.

\begin{tabular}{|c|c|c|c|c|c|c|}
\hline Source & df & Sum of squares & Mean Square & p-value & $\begin{array}{c}\text { Contri. } \\
(\%)\end{array}$ & $\begin{array}{c}\text { Cum. Contri. } \\
(\%)\end{array}$ \\
\hline $\mathrm{F} 1$ & 2 & 21176347 & 10588173 & 0.000 & 84.322 & 84.32 \\
\hline $\mathrm{F} 1 * \mathrm{~F} 3$ & 2 & 527258 & 263629 & 0.000 & 2.099 & 86.42 \\
\hline F4 & 2 & 451643 & 225822 & 0.000 & 1.798 & 88.22 \\
\hline F3 & 1 & 379619 & 379619 & 0.000 & 1.512 & 89.73 \\
\hline $\mathrm{F} 1 * \mathrm{~F} 4$ & 4 & 296815 & 74204 & 0.012 & 1.182 & 90.91 \\
\hline $\mathrm{F} 1 * \mathrm{~F} 2$ & 2 & 146527 & 73264 & 0.038 & 0.583 & 91.50 \\
\hline $\mathrm{F} 2 * \mathrm{~F} 4$ & 2 & 100841 & 50421 & 0.103 & 0.402 & 91.90 \\
\hline $\mathrm{F} 2$ & 1 & 89295 & 89295 & 0.045 & 0.356 & 92.25 \\
\hline $\mathrm{F} 3 * \mathrm{~F} 4$ & 2 & 35945 & 17973 & 0.439 & 0.143 & 92.40 \\
\hline $\mathrm{F} 2 * \mathrm{~F} 3$ & 1 & 7520 & 7520 & 0.557 & 0.030 & 92.43 \\
\hline Error & 88 & 1901857 & 21612 & & 7.573 & 100.00 \\
\hline Total & 107 & 25113668 & & & & \\
\hline
\end{tabular}


Table 5.ANOVAs for Average Ratio of Cell Utilization, disassembly case.

\begin{tabular}{lcllcrr}
\hline \multicolumn{1}{c}{ Source } & df & Sum of squares & Mean Square & p-value & $\begin{array}{c}\text { Contri. } \\
(\%)\end{array}$ & $\begin{array}{r}\text { Cum. Contri. } \\
(\%)\end{array}$ \\
\hline F1 & 2 & 1.018440 & 0.509220 & 0.000 & 42.20 & 42.20 \\
F1*F2 & 2 & 0.219991 & 0.109996 & 0.000 & 9.11 & 51.31 \\
F1*F3 & 2 & 0.201564 & 0.100782 & 0.000 & 8.35 & 59.66 \\
F3 & 1 & 0.142209 & 0.142209 & 0.000 & 5.89 & 65.56 \\
F2 & 1 & 0.141339 & 0.141339 & 0.000 & 5.86 & 71.41 \\
F2*F3 & 1 & 0.023203 & 0.023203 & 0.083 & 0.96 & 72.37 \\
F1*F4 & 4 & 0.000255 & 0.000064 & 1.000 & 0.01 & 72.38 \\
F4 & 2 & 0.000128 & 0.000064 & 0.992 & 0.01 & 72.39 \\
F2*F4 & 2 & 0.000128 & 0.000064 & 0.992 & 0.01 & 72.39 \\
F3*F4 & 2 & 0.000128 & 0.000064 & 0.992 & 0.01 & 72.40 \\
Error & 88 & 0.666167 & 0.007570 & & 27.60 & 100.00 \\
Total & 107 & 2.413551 & & & \\
\hline \multicolumn{7}{c}{$R$-squared $=0.7240$ (adjusted $R$-squared $=0.6644)}$.
\end{tabular}

$R$-squared $=0.7240($ adjusted $R$-squared $=0.6644)$. 
Table 6.ANOVAs for maximum number of intercellular moves.

\begin{tabular}{lrrrrrr}
\hline \multicolumn{1}{c}{ Source } & df & Sum of squares & Mean Square & p-value & $\begin{array}{r}\text { Contri. } \\
(\%)\end{array}$ & $\begin{array}{r}\text { Cum. Contri. } \\
(\%)\end{array}$ \\
\hline F1 & 2 & $1.64 \mathrm{E}+12$ & $8.18 \mathrm{E}+11$ & 0.0000 & 55.88 & 55.88 \\
F1*F3 & 2 & $4.25 \mathrm{E}+11$ & $2.12 \mathrm{E}+11$ & 0.0000 & 14.51 & 70.39 \\
F3 & 1 & $3.45 \mathrm{E}+11$ & $3.45 \mathrm{E}+11$ & 0.0000 & 11.80 & 82.20 \\
F1*F4 & 4 & $2.02 \mathrm{E}+11$ & $5.0527 \mathrm{E}+10$ & 0.0000 & 6.91 & 89.10 \\
F4 & 2 & $1.61 \mathrm{E}+11$ & $8.0423 \mathrm{E}+10$ & 0.0000 & 5.50 & 94.60 \\
F3*F4 & 2 & $4.6543 \mathrm{E}+10$ & $2.3271 \mathrm{E}+10$ & 0.0000 & 1.59 & 96.19 \\
F1*F2 & 2 & 3538851358 & 1769425679 & 0.2260 & 0.12 & 96.31 \\
F2 & 1 & 2804186857 & 2804186857 & 0.1250 & 0.10 & 96.40 \\
F2*F3 & 1 & 2452578482 & 2452578482 & 0.1510 & 0.08 & 96.49 \\
F2*F4 & 2 & 40226703 & 20113351 & 0.9830 & 0.00 & 96.49 \\
Error & 88 & $1.03 \mathrm{E}+11$ & 1168166347 & & 3.51 & 100.00 \\
Total & 107 & $2.93 \mathrm{E}+12$ & & & \\
\hline \multicolumn{7}{r}{-squared $=0.9649$ (adjusted $R$-squared =0.9573). }
\end{tabular}

(a) Disassembly case.

\begin{tabular}{|c|c|c|c|c|c|c|}
\hline Source & $\mathrm{df}$ & Sum of squares & Mean Square & p-value & $\begin{array}{c}\text { Contri. } \\
(\%)\end{array}$ & $\begin{array}{c}\text { Cum. Contri. } \\
(\%)\end{array}$ \\
\hline F1 & 2 & $3.86 \mathrm{E}+12$ & $1.93 \mathrm{E}+12$ & 0.0000 & 67.66 & 67.66 \\
\hline $\mathrm{F} 1 * \mathrm{~F} 3$ & 2 & $1.03 \mathrm{E}+12$ & $5.14 \mathrm{E}+11$ & 0.0000 & 18.02 & 85.68 \\
\hline F3 & 1 & $7.74 \mathrm{E}+11$ & $7.74 \mathrm{E}+11$ & 0.0000 & 13.58 & 99.26 \\
\hline $\mathrm{F} 1 * \mathrm{~F} 2$ & 2 & 2409487460 & 1204743730 & 0.4340 & 0.04 & 99.31 \\
\hline $\mathrm{F} 2$ & 1 & 2157075325 & 2157075325 & 0.2250 & 0.04 & 99.34 \\
\hline $\mathrm{F} 2 * \mathrm{~F} 3$ & 1 & 1146655926 & 1146655926 & 0.3730 & 0.02 & 99.36 \\
\hline Error & 26 & $3.6299 \mathrm{E}+10$ & 1396123046 & & 0.64 & 100.00 \\
\hline Total & 35 & $5.70 \mathrm{E}+12$ & & & & \\
\hline
\end{tabular}

(b) Manufacturing case. 
Table 7.ANOVAs for machine utilization.

\begin{tabular}{lrrrrrr}
\hline \multicolumn{1}{c}{ Source } & df & Sum of squares & Mean Square & p-value & $\begin{array}{r}\text { Contri. } \\
(\%)\end{array}$ & $\begin{array}{c}\text { Cum. Contri. } \\
(\%)\end{array}$ \\
\hline F4 & 2 & 0.792133 & 0.396067 & 0.0000 & 52.00 & 52.00 \\
F1 & 2 & 0.254054 & 0.127027 & 0.0000 & 16.68 & 68.67 \\
F1*F4 & 4 & 0.026927 & 0.006732 & 0.2100 & 1.77 & 70.44 \\
F2 & 1 & 0.017582 & 0.017582 & 0.0510 & 1.15 & 71.59 \\
F3 & 1 & 0.014283 & 0.014283 & 0.0780 & 0.94 & 72.53 \\
F1*F3 & 2 & 0.009536 & 0.004768 & 0.3510 & 0.63 & 73.16 \\
F3*F4 & 2 & 0.006688 & 0.003344 & 0.4780 & 0.44 & 73.60 \\
F2*F4 & 2 & 0.003961 & 0.001981 & 0.6450 & 0.26 & 73.86 \\
F1*F2 & 2 & 0.002495 & 0.001248 & 0.7580 & 0.16 & 74.02 \\
F2*F3 & 1 & 0.000075 & 0.000075 & 0.8980 & 0.00 & 74.03 \\
Error & 88 & 0.39571 & 0.004497 & & 25.97 & 100.00 \\
Total & 107 & 1.523445 & $R$-squared $=0.7403$ (adjusted $R$-squared =0.6842). \\
\hline \multicolumn{7}{r}{}
\end{tabular}

(a) Disassembly case.

\begin{tabular}{lrrrrrr}
\hline \multicolumn{1}{c}{ Source } & df & Sum of squares & Mean Square & p-value & $\begin{array}{r}\text { Contri. } \\
(\%)\end{array}$ & $\begin{array}{r}\text { Cum. Contri. } \\
(\%)\end{array}$ \\
\hline F1 & 2 & 0.037005 & 0.018503 & 0.0000 & 45.24 & 45.24 \\
F2 & 1 & 0.01365 & 0.01365 & 0.0020 & 16.69 & 61.93 \\
F1*F2 & 2 & 0.000568 & 0.000284 & 0.7830 & 0.69 & 62.62 \\
F1*F3 & 2 & 0.000445 & 0.000222 & 0.8260 & 0.54 & 63.17 \\
F2*F3 & 1 & 0.00011 & 0.00011 & 0.7600 & 0.13 & 63.30 \\
F3 & 1 & 0.00003 & 0.00003 & 0.8730 & 0.04 & 63.34 \\
Error & 26 & 0.029988 & 0.001153 & & 36.66 & 100.00 \\
Total & 35 & 0.081797 & & & \\
\hline \multicolumn{7}{c}{$R$-squared $=0.6334$ (adjusted $R$-squared $=0.5065)}$.
\end{tabular}

(b) Manufacturing case. 
Table 8.MANOVA Results.

\begin{tabular}{|l|c|r|r|r|r|r|r|}
\hline \multirow{2}{*}{ Source } & \multirow{2}{*}{$\begin{array}{l}\text { Wilks' Test } \\
\text { (p-value) }\end{array}$} & \multicolumn{2}{|c|}{ Variance contribution (\%) } & \multicolumn{3}{|c|}{ Eigenvectors } \\
\cline { 3 - 8 } & Cost & \multicolumn{1}{c|}{ Size } & \multicolumn{1}{c|}{ Mach. } & \multicolumn{1}{c|}{ Cost } & \multicolumn{1}{c|}{ Size } & Mach. \\
\hline F1 & 0.000 & 63.32 & 82.76 & 5.38 & -0.036 & -0.063 & $\mathbf{1 . 0 3 6}$ \\
\hline F2 & 0.000 & 0.07 & 0.31 & 1.01 & 0.000 & -0.758 & $\mathbf{- 1 . 0 6 9}$ \\
\hline F3 & 0.000 & 1.84 & 0.04 & 1.70 & 0.000 & -0.270 & $\mathbf{- 0 . 5 1 6}$ \\
\hline F5 & 0.000 & 3.54 & 7.96 & 80.17 & -0.054 & -0.201 & $\mathbf{1 . 5 7 0}$ \\
\hline F1*F2 & 0.000 & 0.11 & 0.00 & 1.24 & -0.092 & $\mathbf{- 1 . 0 5 8}$ & 0.184 \\
\hline F1*F3 & 0.000 & 2.58 & 0.00 & 1.65 & -0.006 & -0.440 & $\mathbf{0 . 9 3 8}$ \\
\hline F1*F5 & 0.000 & 3.96 & 0.01 & 1.93 & -0.012 & -0.275 & $\mathbf{0 . 6 9 5}$ \\
\hline F2*F3 & 0.146 & 0.04 & 0.02 & 0.07 & 0.001 & $-\mathbf{- 0 . 7 8 2}$ & -0.475 \\
\hline F2*F5 & 0.970 & 0.04 & 0.07 & 0.08 & -0.006 & -0.343 & $\mathbf{- 0 . 5 1 7}$ \\
\hline F3*F5 & 0.000 & 0.44 & 1.36 & 2.01 & -0.087 & -0.267 & $\mathbf{1 . 1 5 3}$ \\
\hline Error & & 24.05 & 7.35 & 4.76 & & & \\
\hline
\end{tabular}




\section{LIST OF FIGURES}

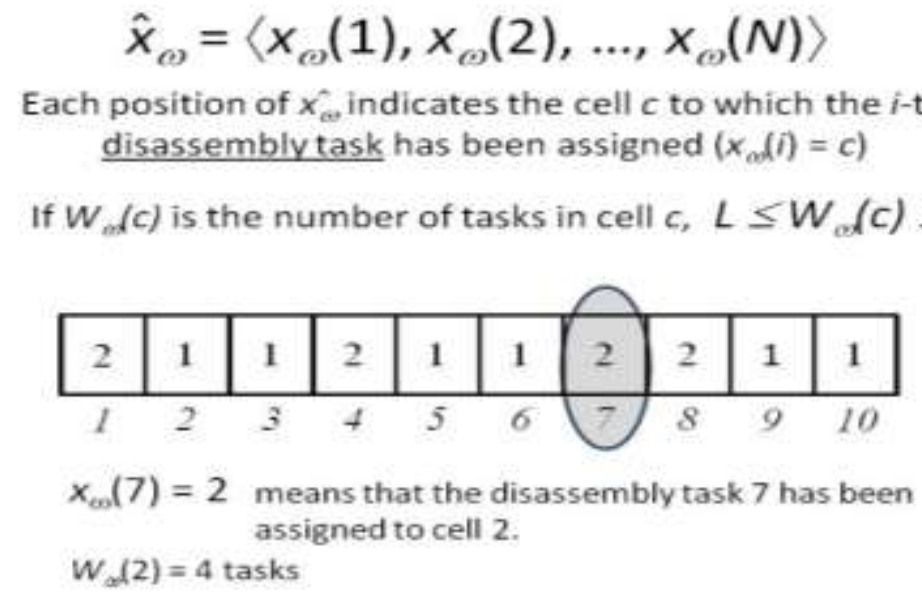

Figure 1. Solution coding

Note: This figure shows a possible representation of a solution with two cells for a problem with five parts (where each one requires two operations) therefore there are 10 tasks.
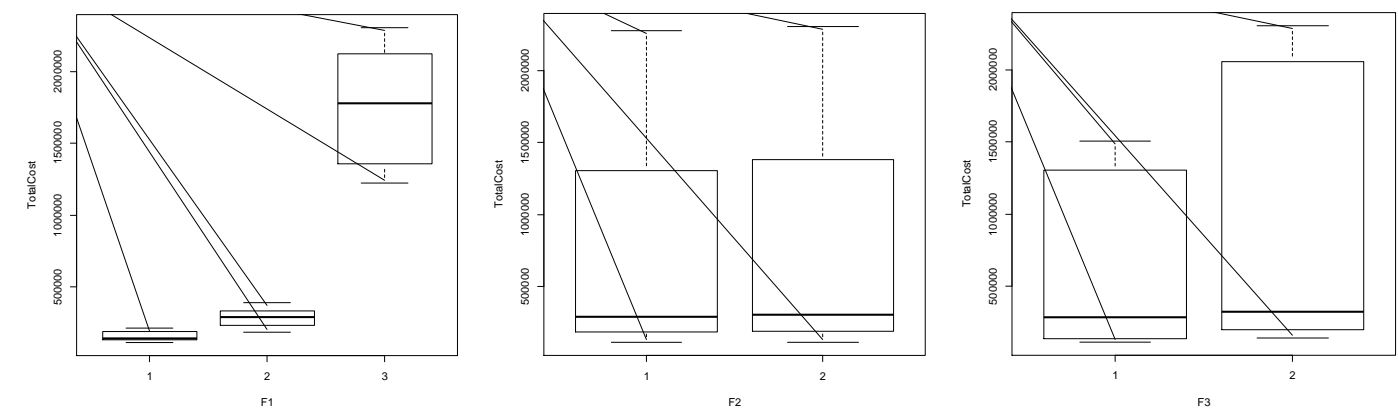

Figure 2. Boxplot for Total Cost: manufacturing case.

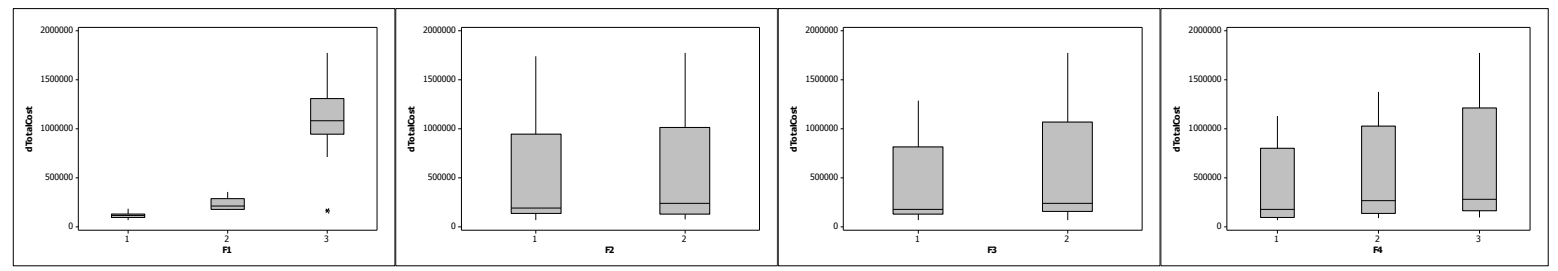

Figure 3. Boxplot for Total Cost: disassembly case. 


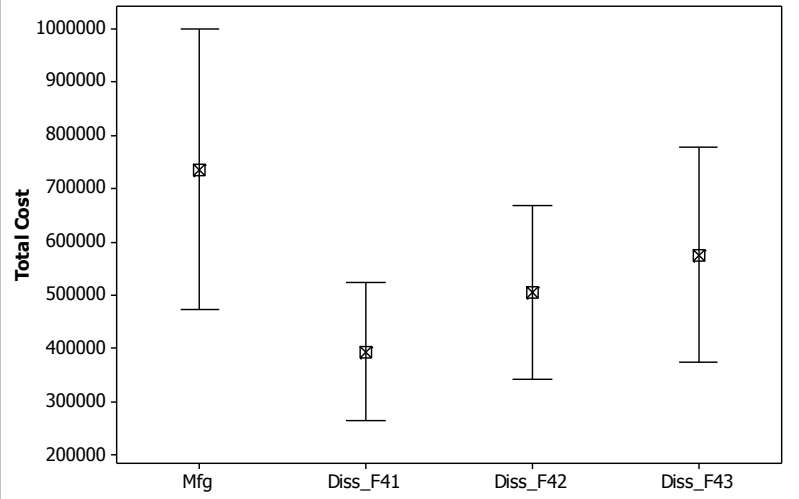

Figure 4. Confidence interval at $95 \%$ for the total cost between manufacturing and the three disassembly environments. 